\title{
Designing Creative Destruction
}

\author{
Ashley Hall ${ }^{\mathrm{a}}$ \\ ${ }^{a}$ Royal College of Art \\ *ashley.hall@rca.ac.uk
}

\begin{abstract}
This research aims to make a contribution in the context of design thinking at a global cultural scale and specifically how design methods are a feature of the homogenising and heterogenising forces of globalisation via creative destruction. Since Schumpeter's description of economic innovation destroying the old and creating the new, a number of other interpretations of creative destruction have developed including those driving cultural evolution. However a design model showing the impact of different types of design method on cultural evolution can develop an understanding on a more systemic level from the medium to longer term impact of new designs that homogenise or increase the differences between various cultures. This research explores the theoretical terrain between creative destruction, design thinking and cybernetics in the context of exchanging cultural influences for collaborative creativity and concludes with an experiment that proposes a feedback loop between ubiquitising and differentiating design methods mediating cultural variety in creative ecosystems.
\end{abstract}

Keywords: Creative Destruction; Design Thinking; Cybernetics; Globalisation

\section{Introduction}

The design thinking developed in this paper is drawn from a number of publications (Hall, 2013; Hall, 2011; Hall, 2015; Hall, forthcoming 2016) and keynote speeches delivered by the author (Hall, 2009; Hall, 2015). Primarily it seeks to bring together a more coherent set of thoughts on the relationship between design and creative destruction and in particular two important elements of this relationship. The first is a meta-level attempt to theorise on how the use of homogenous (ubiquitising) and heterogeneous (differentiating) design methods can affect the future development of creative cultures via design initiatives for the generation of products and experiences. The second is to attempt to explore the connections between the concepts of creative destruction and design thinking on globalisation to investigate if design practice can tangibly engage to develop more positive variations in cultural diversity. 
Design is a key agent in mediating the impact of globalisation due to its direct effect on products and services experienced by consumers and its role in increasing global communications. For example franchises designed for global food and consumer goods outlets have for the last few decades appeared in larger number of cities in more diverse corners of the world, offering on the one hand a reliable unified experience but on the other homogenising choice and reducing diversity and affecting local cultural traditions. On the other hand it could be argued (Hall, 2015) that ubiquitisation has also democratised choice and removed what has in the past been seen as unwelcome colonial imprints that some have viewed as being contrary to the future good of particular communities.

Design and geographically Liberated Difference (Hall, 2011) was a concept initially exploring the role that design could take in extracting and communicating cultural influences through collaborative design activity in local communities. This line of thought was developed through a practice based doctoral thesis (Hall, 2013) that linked frameworks of global cultural anthropology to visualising the exchange of cultural material that impacted on physical features in designed products leading to a theory of 'translocated making' in collaborations between crafts people and industrial designers (Hall Forthcoming 2016). A recent publication (Hall, 2015) pursued this line of thinking in terms of the ubiquitisation of global mass produced products and began to unpack the design influences that drive homogeneous and differentiated designs for a global mass market. In parallel to the practice based research, two keynote speeches on city design innovation in Shenzhen (2009) and Taipei (2015) initiated a line of thought around the future development of design innovation cultures at a city scale. Furthermore this led to thoughts on how urban cultures are nurtured and what sets them apart from the other competing global cities who are also experiencing similar homogenising tendencies.

What unites the diverse scales between local practice based design projects to city scale creative ecosystems is to ask whether design thinking can have a meaningful impact in directing more positive and equitable results via cultural change. At this point it's worth clarifying that a 'culture' is used as an open term here to recognise both the traditional geolocated, alongside emerging transnational and the increasingly fragmentary and spatially liberated cultural affinities that are emerging as products of globalisation.

In moving towards a design exploration of creative destruction it is necessary to conduct a review of the term that can provide us with a useful historical perspective of how thinking has evolved and moved into new disciplinary fields.

\section{Creating Destruction}

Joseph Schumpeter described creative destruction as an economic innovation model that impacted on the business cycle by destroying the old in order to make the new (Schumpeter, 1943). Although previous examples of technology led disruptive innovation through industrialisation including the telegraph and the railroad had been noted previously, Schumpeter can be credited with unifying the concept and describing its impact at an 
economic level. Schumpeter's model directly contradicted the 'guiding hand' of Adam Smith's (1776) economic theory that gave so much support to the idea of a nation state directing future economic matters. Instead, industries, products and innovations were the disrupting forces in Schumpeterian economics, a series of variables much harder to govern at national level and a situation that continues to this day as globalised corporations increasingly outpace national governments in their ability to shape the lives of citizens. The impact of Adam's Smith's economic model can be associated as much with conservative homogenising tendencies as Schumpeter's with radical disruptions.

The impact of disruptive innovations in economics can also be allied to Appadurai's concept of disjunctions in his suffixscapes framework (Appadurai, 1990). A theory that is useful in supporting discussions on how inequalities in cultural material flows affect different types of recipient by creating impactful 'disjunctions' or events that divert previously expected futures. Disjunctions are the effects of disruptions to cultures sometimes driven via creative destruction where new technologies change material and behavioural worlds. As explored by the author (Hall, 2013; Hall Forthcoming 2016), the Suffixscape framework can be connected to direct tangible interactions between individuals and can show that global concepts can have local impacts in the production of artefacts. Suffixscapes comprise a series of inter-operable landscapes of media, technologies, finances, ethnographics and ideas, and can be described as the cultural impact points of creative destruction.

Tyler Cowen (2002) positions creative destruction into a cultural context by describing a broad field of cultural interactions creating differences that can enhance or subtract from an existing cultural group. In many ways this relates to Appadurai's suffixscapes (1990) in its proposition of non-linear relationships for information exchange. Cowen deconstructs the process of cultural exchange to expose the potential benefits of increasing the differences that arrive from other geographies and how these can challenge or support the future cohesion of cultural groups. Where there is loss in some areas, others have greater gains. $\mathrm{He}$ states that all successful cultures become hybrids and that their future survival is based on their ability to evolve and absorb new differences. The patronising idea of 'museum cultures', a state where groups are preserved for future study is roundly demolished. In addition the assumption of global homogenisation where cities across the world have the same fast food chains, clothing stores and mobile phone concessions is challenged by the volume, density and diversity of cultural interactions that are negotiated via each culture's value filtering system. It is argued that this scenario accelerates diversity rather than producing homogenisation.

\section{Cowen writes:}

The benefits of cultural exchange usually have come from dynamic settings in great imbalance, rather than from calm or smoothly working environments...Does trade in cultural products support the artistic diversity of the world, or destroy it? Will the future bring artistic quality and innovation, or a homogenous culture of least 
common denominator? What will happen to cultural creativity as freedom of economic choice extends across the globe? (Cowen, 2002, p. 7)

From a similar direction the philosopher Kwame Appiah (2006) offers a viewpoint promoting cultural contamination as a necessary and present ingredient to evolve and sustain healthy cultures. He describes the concept of cultural contamination as a vital and necessary force of interaction and enrichment for future development and describes the evolutionary pace of cultural contamination:

Living cultures do not, in any case, evolve from purity into contamination; change is more a gradual transformation from one mixture to a new mixture, a process that usually takes place at some distance from rules and rulers, in the conversations that occur across cultural boundaries. (Appiah, 2006, p. 6)

Appiah contends that all cultures are hybrids by nature and that successful survival depends upon continued refreshment from external sources that helps cultural behaviours to develop and adapt to evolving situations. This contrasts with the conservative view of preserving cultures intact and attempting to reduce the external influences that are deemed to be harmful. In essence this view promotes the isolation of cultures from what are seen as contaminating influences that may dilute certain practices. The nostalgia for pristine cultures that are observed and controlled externally could be seen as both colonialist and undemocratic in limiting the choices and freedoms enjoyed by others. Appiah sees this approach as increasing the risk of cultural collapse and asymmetrical disconnection.

Bridging the gap between design research that has brought important insights into cultural influences and the value to industrial design in the emerging field of cultural exchange research (Lin, 2007; Moalosi, 2007; Yao, 2015) and transnational cultural frameworks and concepts (Appadurai, 1990; Cowen, 2009; Fiss, 2009) is the practice of selecting design methods. Choosing different design methods can promote either homogenising or heterogeneous effects on different cultures.

Savov (2015) amongst others (Hall, 2015) have been highly critical of the ubiquitising tendencies in mass produced consumer products and has highlighted the dangers of:

While their approaches and economic models differ, the near future that Apple, Google, and Microsoft perceive is remarkably similar. Ask any of them about the smartphone of tomorrow and you'll get an answer that involves a grid of icons, an app store, a great display and camera, and broadly the same industrial design proportions and philosophy (Savov, 2015)

It is clear that in this example homogenizing design strategies are being selected that produce minimal ubiquitous products with very similar aesthetics, operating systems and technologies with an effect that reduces variety and competition for consumers (E.G Apple, Samsung, LG, Huawei smartphones as above). Conversely it could be argued that reducing cultural imprints allow the production of more units to cover broader markets lowering prices and democratising choice alongside reducing negative cultural impact from strong 
influences. Whichever versions of these realities play out it is clear that design is a major factor in mediating the forces of creative destruction.

At this point we can ask; What are the strategic meta-level roles that design thinking can play in being a positive agent of change in the enormous flow of information and cultural expressions that are exchanged globally on a day-to-day basis? It would seem that a good starting point is to look at design methods and begin to ask where and when should we consider deploying different types of methods depending on whether we can foresee that ubiquitous or differentiated outcomes are most desirable. Can design thinking and design methods affect the balances and tipping points of ubiquitising and differentiating forces in the circulation of cultural exchange?

\section{Requisite Variety}

One of the key concerns at the centre of this discussion is the quota of variety that exists in any creative ecosystem and how that affects design practice (Hall, 2015). The amount of variation in a system affects the number of possible solutions of the combination of its elements and also directly relates to the potential for creative destruction impact. It follows that for example if we are looking to develop creative communities that are resilient and able to generate responses that adapt to future global disruptions either human-made or human-caused then this is a highly desirable capacity to encourage. Whether it is developments in biotech products, nanotechnology or environmental tipping points, the future requirements of creative and responsive innovation will need more, not less variety in the potential number of solutions we can generate. However developments in design education (Inella 2011; Hall, 2015), design strategies from design studies of global massmanufacturers (Savov, 2015) and 3d printing systems are producing ubiquitising tendencies that encourage global conformity, lowest common denominator solutions, culture free products and other impacts that are causing serious concern for the amount of creative variety on offer. In addition the creative communities of our global cities are so successful at rapidly sharing ideas and insights that it is increasingly difficult to be surprised or delighted due to the decreasing differences encountered in city experiences. The impacts of these developments will surely rebound up to national and transnational economic models as design and innovation capacity continues to rely on creating more variety as a measure of success and future value.

W. Ross Ashby developed the concept of requisite variety (Ashby, 1958) to describe the potential number of given states of any system. Later on this was developed in second order cybernetics to offers us a potential way of modelling a set of relationships in terms of feedback loops where the observer is in the interaction and recognised as being capable of affecting the information flow in the system (observing the observer who may or may not be oneself). Second order cybernetics is crucial in recognising the observer as an active participant in the relations between human and machine and that the relationship is recursive and interdependent. The 'states' that we are interested in here are the number of 
different design solutions that may be generated within a particular design culture and the impact of those solutions on recipients or users of the designs either within that culture or globally. Design methods in this case can be selected for a number of reasons, one of which includes approaches that could be said to increase the variety between design solutions on the market and those that decrease variety. Variety in this instance includes the functions of the products themselves and the features, affordances, material choices, colours, aesthetics, technologies and any other attributes with the capacity for generating differences to the lives of users.

\section{Designing Creative Destruction}

What follows is an experiment to try and bring together a way to understand how cultural materials flow and influence cultures in an economy geared towards greater variety for future resilience. Glanville's extensive discussions and philosophies relating second order cybernetics to reflective and practice based design (E.G. Glanville, 1994) are important in this respect and especially his discussion of 'knowledge for' future action and possibilities rather than 'knowledge of' past actions and events (Glanville, 2005). The intention is that the model sketched out here may be useful for considering future possibilities when organisations, institutions and policies consider projects and initiatives in terms of how they can impact on creative diversity for cultural benefit. In order to develop a model of designing creative destruction it is important to set out the context for variability and the operational scale within which this may occur. Two distinct sets of variables will be required to reflect the needs of creative ecosystems of organisations and individuals as well as the effects of those individuals through manufactured products, designs and systems released into the public sphere. The model will therefore need to cope with the increase or decrease of variety through the effects and influences of manufactured products and design in circulation and increase or decrease the variety of creative solutions in circulation. In addition the model will also need to reflect the different scales that could be operational in this sphere, for example from the individual small-scale local collaborations between designers and craftsmen through to city scale decisions. For example the best area to set up an innovation park, technology centre or creative hub.

It was suggested earlier in this discussion that ubiquitised and differentiated design outcomes can vary the amount of creative destruction in circulation. Whilst it may sound relatively simple in theory to list practices that homogenise and practices that differentiate, the reality is more complex. For example a new communications product may be designed with a very minimal black geometric case yet contain disruptive technologies that adversely affect the user on a behavioural level. The aesthetic impact of the product may be negligible but the technology disruption may be very high. The physical effect is ubiquitous with a low potential impact for change but the function is disruptive and differentiated with a high potential impact for change. Going forwards a 'design' is considered in all of its potential impacts on cultural influence both in terms of technologies, experiences, new behaviours, aesthetics and other factors. 
In order to consider how design methods can change the amount of creative destruction in an ecosystem a number of methods and approaches are listed. These are intended as examples for populating the model but it is recognised that they are not an exclusive or exhaustive list and that their effects will not be limited to exclusively homogenise or generate heterogeneous differences in outcomes. Examples of design methods and approaches that can encourage a homogenous result include: minimal design, ubiquitous design, focus groups, user-centred design. Examples of design methods, tools and approaches that can encourage a heterogeneous result include: experimental design, critical design, brainstorming, translocated making and cultural transfer.

Figure 1. introduces a concept model for designing creative destruction as a feedback loop regulating the selection of design methods for more equitable cultural development. In order to aid description we will begin with the designers or organisations who select methods, tools and approaches that produce more or less variety in the cultural ecosystem due to their homogenising or differentiating effects. The method examples above have been used to populate the model but there are many others that could also be used with varying effects. In some situations combinations of methods may need to be used for more complex projects or when tacking nested or complex wicked problems. The impacts are assessed at a point where a decision is made to increase variety or decrease variety and can be based on a number of factors affecting cultural development from similarity to existing cultural typologies suggesting homogenisation through to the need to cope with new technologies or other impacts suggesting greater differentiation. 


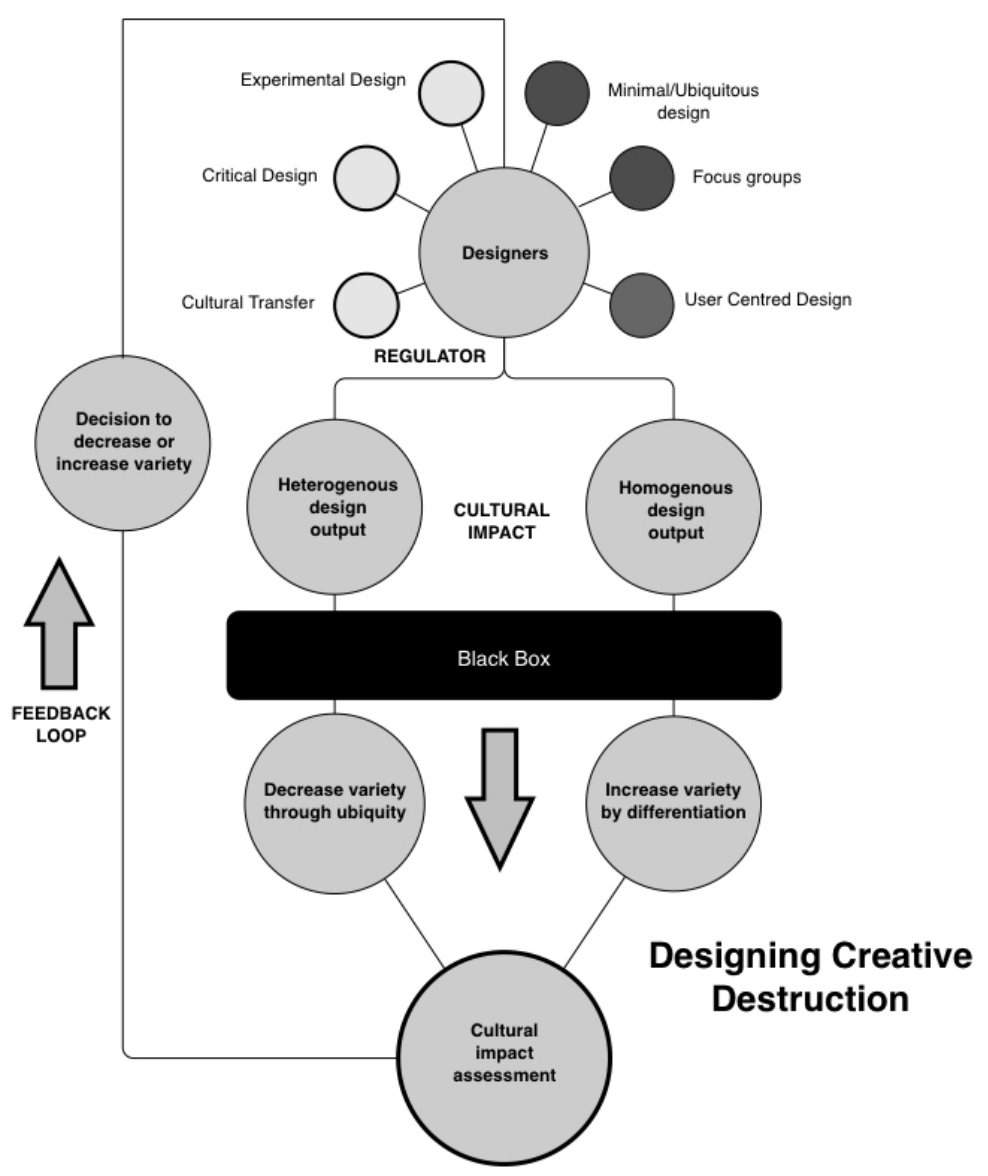

Figure 1: Model for regulating variety for designing creative destruction

The aim of producing this model however is not to propose a system that can cope with all the myriad complexities of a system of cultural influence in the full flow of global information exchange with all of its many media platforms, actors and agenda's, but to begin building the main blocks associated with the system to ask what can be achieved and what is required from the parts in order to ask if meta level design approaches can bring value. In practice when considering a scenario where greater cultural variety is required in order to be more competitive or include a wider section of users a decision would be made to select design methods and approaches that generate more differences. This could take place in projects between collaborating designers from different cultures up to city planners deciding which approaches would increase creativity and innovation in different urban sectors.

Criticisms of the model have to take into account Schumpeter's assertion that capitalist systems of creative destruction cannot be analysed in real time and instead can only be assessed retrospectively:

We are dealing with a process whose every element takes considerable time in revealing its true features and ultimate effects, there is no point appraising the performance of that process ex visu of a given point of time...since we are dealing with an organic process, analysis of what happens in any particular part of it-say, in 
an individual concern or industry-may indeed clarify details of the mechanism but is inconclusive beyond that. (Schumpeter, 1943, p83)

However Schumpeter's concerns are with exploring the real nature of a capitalists system and its macro economic effects whereas the discussion here is around cultural influence on a number of scales from the micro to the macro and so it is not clear that the limitations described above continue to stand, especially in light of new digital means of researching and gathering evidence across networks. The central issues concerns if we can ever form an understanding of cultural exchanges that can affect the requisite variety of a creative ecosystem in a timescale such that it can produce beneficial directed results.

Essentially the inputs are the selection of homogenising or differentiating design methods and the outputs are cultural changes. Another concept from cybernetics can be of assistance here, the black box (Ashby, 1956). Principally this can be summarised as a method of dealing with a complex or unknown system that we can interact with via its inputs and outputs. While we cannot see the mechanism inside the box we can develop an understanding of how to interact with it via the differences between inputs and outputs. If we use an increase number of homogenising design methods the amount of variety will lower and it may be easier to understand the mechanism of cultural exchange, the box will become more transparent or to use the cybernetic term 'whitened' (Glanville, 2002). However if we increase the differences then we also increase the complexity and the box will be 'darkened'. While this effect may not reveal the underlying mechanisms it may well indicate the state or balances of ubiquitising or differentiating methods being used.

\section{Conclusions}

This paper has reviewed the relationship between design and creative destruction and speculated on an experimental system for understanding if meta level design thinking can bring together a model for how creative destruction can be designed to enhance the evolution of cultures through mediating in the choice of design methods selected for a variety of output scales from individual products to urban and city scales. It can provide support for more strategic choices in terms of the types of design approaches and design methods selected in terms of cultural impact.

Variety is at the core of the rationale for the selection of different design methods and while these relationships have been sketched in outline there remains significant work to develop and explore these aspects in more detail. In terms of regulation more work is required in order to understand what sort of agencies, whether governments, institutions, private companies and design organisation can have a role to play. There may well be systemic complexity beyond that which is governable and this is represented by the complex cultural exchanges and influences that take place in the black box. The politics of increasing or decreasing diversity are likely to be a point of contention, especially in light of the fact that more diverse groups are more likely to challenge authority hence there could be a tendency to reduce variety in order to increase the tendency to conform. One key question that 
remains is how can we assess the future amount of requisite variety needed in such a system, especially as future impacts may be disruptive and not forecastable on an on-going basis from historical models. The likelihood of future global climactic tipping points being breached are a clear scenario when more diverse problem solving skills maybe be required in a short timeframe. A more complex situation is the rapidly increasing urbanisation of global citizens, mass migrations and the difficulties in maintaining cultural belief systems in new locations alongside the proliferation of 'everything everywhere' digital networks.

If we can find ways to see the 'whitening' or 'darkening' of the black box of cultural impacts and create a barometer of variation influencing cultural systems then this could be highly beneficial, even if it is applied to specific product categories like smartphones which are increasingly criticised for their ubiquity (Hall, 2015; Savov, 2015). Elements of mechanisms designed to detect this state may well exist across a number of fields including design thinking, social sciences, anthropology and economics and require an interdisciplinary effort to draw these together. Since the formation of Schumpeterian economics our understanding of capitalist forces of competition, market supply and disruptive technologies alongside quantitative and qualitative methods of assessing impacts have grown substantially. However the design impact goes beyond disruption and also includes the value of homogenization where value can come from reducing variety in a system and we could draw an analogy between Adam's economic model of the guiding hand and Schumpeter's model of creative destruction as both seem to align with a system that attempts to govern the amount of variety in circulation for creative practices and cultural economic benifit.

In terms of cybernetics the projecting and assessing of cultural impact may possibly fall into the sociocybernetic approach of scaling to a social set of feedback loops described by (Felix and Van Der Zouwen, 1991) or Stafford Beer's viable system model (1984) is challenging from the point of view of how designing creative destruction via varying the use of homogenizing or heterogeneous design methods is meeting the demands of surviving in a challenging current or future creative cultural environment. Third order cybernetics (Glanville, 2002) may solve the issue of needing to observe the outcomes of cultural change and replace it with an acting/observer to cope with the impacts by selecting different design methods or approaches in a more tacit or reflexive manner.

It is hoped that the thinking initiated here positions design thinking as a key mediator in the space between a number of disciplines including design, economics and anthropology in steering our future efforts in shaping the worlds between socio-economic systems and cultural evolutions that provide us with the creative variety we need to develop resilient societies with diverse cultural expressions and a capacity to design and innovate to meet future global sustainability challenges.

\section{References}

Appiah, K. A. (2006). The case for contamination. New York Times, Sunday Magazine, Jan 1. 
Ashby, W. (1956) An introduction to cybernetics, London: Chapman \& Hall, chapter 6: The black box, pp. 86-117.

Ashby, W.R. (1958,) Requisite Variety and its implications for the control of complex systems, Cybernetica (Namur) Vo1 1, No 2, 1958.

Appadurai, A. (1990). Disjuncture and difference in the global cultural economy. Theory, Culture and Society, 7, 295-310.

Beer, Stafford. (1984) "The viable system model: Its provenance, development, methodology and pathology." Journal of the operational research society, pp7-25.

Cowen, T. (2002). Creative destruction: How globalization is changing the world's cultures. USA: Princeton University Press.

Fiss, K. (2009). Design in a global context: Envisioning postcolonial and transnational possibilities. Design Issues, 25(3), 3-10.

Felix, G. and van der Zouwen, J., (1991) "Cybernetics and social science: Theories and research in sociocybernetics." Kybernetes 20.6, pp81-92.

Glanville, R., (1994), Variety in Design, Systems Research, 11(3).

Glanville, R. (2002). Second order cybernetics. Encyclopaedia of Life Support Systems.

Glanville, R., 2005, The Unthinkable Doctorate: Brussels, Design Prepositions. Cyberethics Research, American Society of Cybernetics, UK and Australia.

Hall, A. \& Barker, T. (2011). Design and geographically liberated difference. Design Principles and Practice: An International Journal, 5(3), 507-516.

Hall, A. (2011). Experimental design: Design experimentation. Design Issues, 27(Number 2, Spring 2011), 17-26.

Hall, A. (2013),'Translocated making in collaborative experimental design projects', PhD thesis, University of Technology Sydney.

Hall, A. (2015). Ubiquitous Tendencies: Cultural Inspirations and the Future of Industrial Design for Global Mass Market, Korean Society of Design Science (KSDS).

Hall, A. (Forthcoming 2016), The Emergence of Translocation in The Encyclopaedia of Asian Design Vol 4. Bloomsbury (Berg), London.

Innella, G. Rodgers, Spencer, N., \& Bohemia, E. (2011). Examining the physical to visual shift in how we now experience designed objects. In IASDR.

Lin, R. (2007). Transforming Taiwan aboriginal cultural features into modern product design - A case study of cross cultural product design model . International Journal of Design, 1(2).

Moalosi, R. (2007). The impact of socio cultural factors upon human centred design in Botswana. Thesis, Australia: Queensland University of Technology.

Savov, V., (2015). Never mind the future, we need more diverse visions of the present, The Verge, Retrieved 21/9/2015 http://www.theverge.com/2015/9/18/9351197/apple-google-microsofttech-innovation-uniformity

Schumpeter, J. (1943), Captalism, Socialism, Democracy, Routledge, London and New York.

Smith, A. (1776). An Inquiry into the Nature and Causes of the Wealth of Nations, 1st edn 1776, vol. II of The Glasgow Edition of the Works and Correspondence of Adam Smith, edited by RH Campbell, AS Skinner and WB Todd." 1976.

Yao, W., (2015) Approaching Chineseness: Investigating the cultural transfer of behavioural factors in and through Chinese industrial design, PhD thesis, Royal College of Art. 
About the Author:

Ashley Hall is Professor of Design Innovation at the Royal College of Art's Innovation Design Engineering programme where he is head of research. He researches in a number of design and innovation fields including design thinking, experimental design, future of making, cultural transfer and translocated making. 